\title{
Characterization of Benzene Samples Taken from Different Areas in Sudan Using Laser Induced Breakdown Spectroscopy (LIBS)
}

\author{
Sufyan Sharafedin Mohammed Dawod“, Nafie A. Al Muslet, Ahmed Abubaker Mohamed Taher \\ Department of Physics, College of Science, Sudan University of Science and Technology, Khartoum, Sudan \\ Email address: \\ sufyansharaf@gmail.com (S. S. M. Dawod),mnmfa2008@yahoo.com (N. A. Al Muslet), ahmedabubaker5555@gmail.com (A. A. M. Taher) \\ ${ }^{*}$ Corresponding author
}

To cite this article:

Sufyan Sharafedin Mohammed Dawod, Nafie A. Al Muslet, Ahmed Abubaker Mohamed Taher. Characterization of Benzene Samples Taken from Different Areas in Sudan Using Laser Induced Breakdown Spectroscopy (LIBS). American Journal of Modern Energy.

Vol. 6, No. 4, 2020, pp. 77-83. doi: 10.11648/j.ajme.20200604.11

Received: May 28, 2020; Accepted: June 20, 2020; Published: November 4, 2020

\begin{abstract}
Protecting the environment from all types of pollution is a key issue, especially air pollution in particular, because of that, the ecosystem as a whole is under threat. Sudan is one of the vast African countries, covering an area of about one million square miles, and suffering from air pollution. One of the causes of this pollution is the large number of cars and factories that use gasoline. Car exhausts and factory nozzles emit kinds of gases such as carbon monoxide, lead, sulfur and other gases that contribute to air pollution. Due to the large volume of use of gasoline in cars and factories, the low quality of purification and treatment leads to the production of gases that are extremely harmful to the environment. Therefore, it is necessary to examine and determine the components of benzene used in Sudan. The technique of LaserInduced Breakdown Spectroscopy was used to characterize the benzene samples taken from different regions, and the results showed that they contain carbon and hydrogen that are the main component of benzene, in addition many elements harmful to the environment such as $(\mathrm{Pb}, \mathrm{Hg}$ and $\mathrm{Zn})$, which can cause cancer diseases, and can also cause great harms to animals, plants and soil.
\end{abstract}

Keywords: Laser Induced Breakdown Spectroscopy, Benzene, Protecting the Environment, Pollution, Sudan

\section{Introduction}

Many scientific, military, medical and commercial laser applications have been developed since the invention of the laser in 1958. The coherency, high monochromaticity, and the ability to reach extremely high powers are all properties which allow for the specialized applications [1].

Laser analysis based on optical detection method is called laser spectroscopy. Spectroscopy involves stimulating as ample and the analyzing the resultant spectrum in the range of electromagnetic radiation emitted or absorbed by the sample that makes Spectroscopy is a vital as an analytical tool [2].

The Laser Induced Breakdown Spectroscopy (LIBS) technique is based upon the analysis of the atomic emission generated close to the surface sample. The emission is observed once a laser pulse is focused on the sample surface, where the very high field intensity initiates an avalanche ionization of the sample elements, giving rise to the so-called breakdown effect, the formation of the plasma Occur when the focused laser achieves a certain threshold for optical breakdown, which generally depends on the environment and the target material $[3,4]$.

Laser-induced break down spectroscopy, or LIBS, has been advancing significantly over the last decades. It can be used for characterizing solids, liquids and gases and can return results rapidly, with very little damage to the sample. Not only that, but also the samples can be analyzed from a distance away from the source of the (LIBS). Unlike some analytical tools that require samples being brought to a lab. For example, LIBS is being used to detect surface contaminants in a few nuclear reactors around the world, in this case The lasers used in these systems is located several meters away from the reactor 
surface and yet is still able to function effectively. These systems keep most of the instrumentation behind shielding material, with only a mirror and a lens (which is used to steer and focus the laser beam respectively) exposed to the nuclear radiation [3].

LIBS is a very useful technique, Through it the elements that formed materials can be recognized and identified easily and fast way, under any atmosphere. It requires very small or even no sample preparation.

It is very sensitivity technique and capable of producing low limits of detection (LOD). LIBS can be considered as a non-destructive technique since it involves negligible amount of mass for analysis (micro). LIBS can, in principle, be applied in any atmosphere, even in hostile environments, therefore, it is very handy for insitu analysis and in-process monitoring of the product [5].

\section{Materials and Method}

\subsection{Materials}

Materials used in this work as samples were three types of benzene. It was collected from different stations in Sudan.

Benzene is an organic chemical compound with the molecular formula $\mathrm{C}_{6} \mathrm{H}_{6}$. Its molecule is composed of 6 carbon atoms joined in a ring, with 1 hydrogen atom attached to each carbon atom. because its molecules contain only carbon and hydrogen atoms, benzene is classed as a hydrocarbon as shown Figure 1.

Benzene is a natural constituent of crude oil, and is one of the most elementary petro chemicals. Benzene is an aromatic hydrocarbon and the second [n] -annulene ([6]-annulene), a cyclic hydrocarbon with a continuous pi bond. It is sometimes abbreviated $\mathrm{Ph}-\mathrm{H}$. Benzene is a color less and highly flammable liquid with a sweet smell. It is mainly used as a precursor to heavy chemicals, such as ethyl benzene and cumene, which are produced on a billion kilogram scale. Because it has a high octane number, it is an important component of gasoline, composing a few percent of its mass. Most non-industrial applications have been limited by benzene's carcinogenicity.

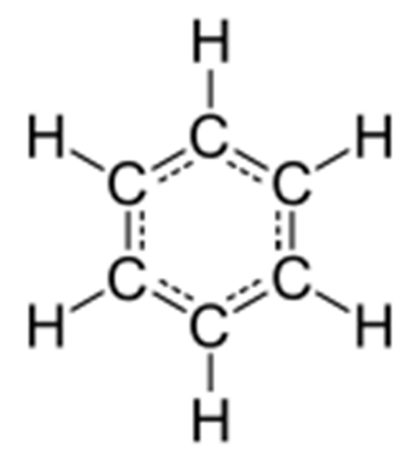

Figure 1. Ring Formula of Benzene.

The empirical formula for benzene was long known, but it's highly poly unsaturated structure, with just one hydrogen atom for each carbon atom, was challenging to determine.
Archibald Scot Couperin 1858 and Joseph Loschmidt in 1861 [5]. Suggested possible structures that contained multiple Double bonds or multiple rings, but too little evidence was then available to help chemists decide on any particular structure.

Benzene is classified as a carcinogen, which increases the risk of cancer and other illnesses, and is also a notorious cause of bone marrow failure. Substantial quantities of epidemiologic, clinical, and laboratory data link benzene to a plastic anemia, acute leukemia, bone marrow abnormalities and cardiovascular disease [6, 7]. The specific hematologic malignancies that benzene is associated with include: acute myeloid leukemia (AML), aplastic anemia, myelodysplastic syndrome (MDS), acute lymphoblastic leukemia (ALL), and chronic myeloid leukemia (CML) [8].

The American Petroleum Institute (API) stated in 1948 that "it is generally considered that the only absolutely safe concentration for benzene is zero" [9]. There is no safe exposure level; even tiny amounts can cause harm. The US Department of Health and Human Services (DHHS) classifies benzene as a human carcinogen. Long-term exposure to excessive levels of benzene in the air causes leukemia, a potentially fatal cancer of the blood-forming organs. In particular, acute myeloid leukemia or acute nonlymphocytic leukemia (AML \& ANLL) is not disputed to be caused by benzene [10]. IARC rated benzene as "known to be carcinogenic to humans" (Group 1).

As benzene is ubiquitous in gasoline and hydrocarbon fuels that are in use everywhere, human exposure to benzene is a global health problem. Benzene targets the liver, kidney, lung, heart and brain and can cause DNA strand breaks, chromosomal damage, etc. Benzene causes cancer in animals including humans. Benzene has been shown to cause cancer in both sexes of multiple species of laboratory animals exposed via various routes [11].

\subsection{Instrumentation}

The experimental set up used in this work is shown in figure 2 and schematically are depicted in figure 3.

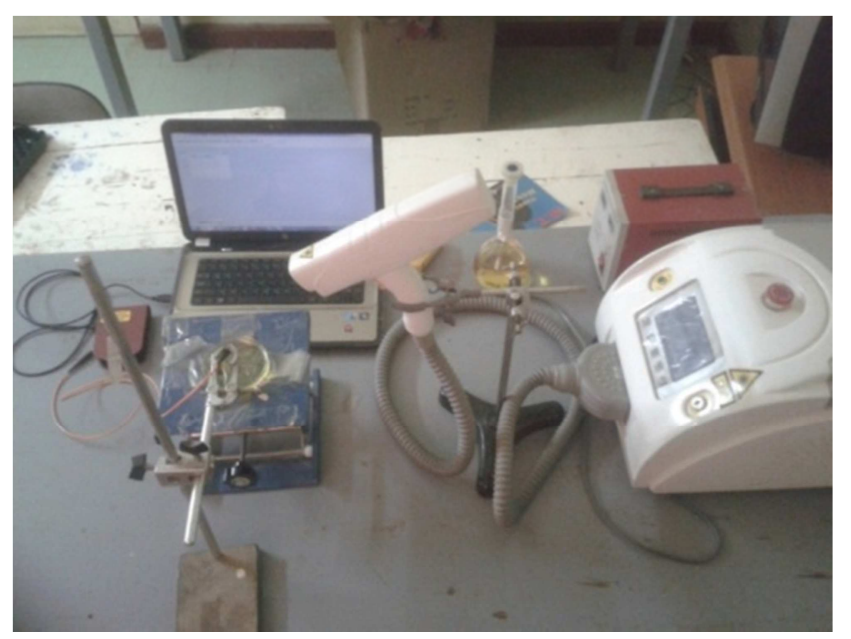

Figure 2. The Experimental Set up. 


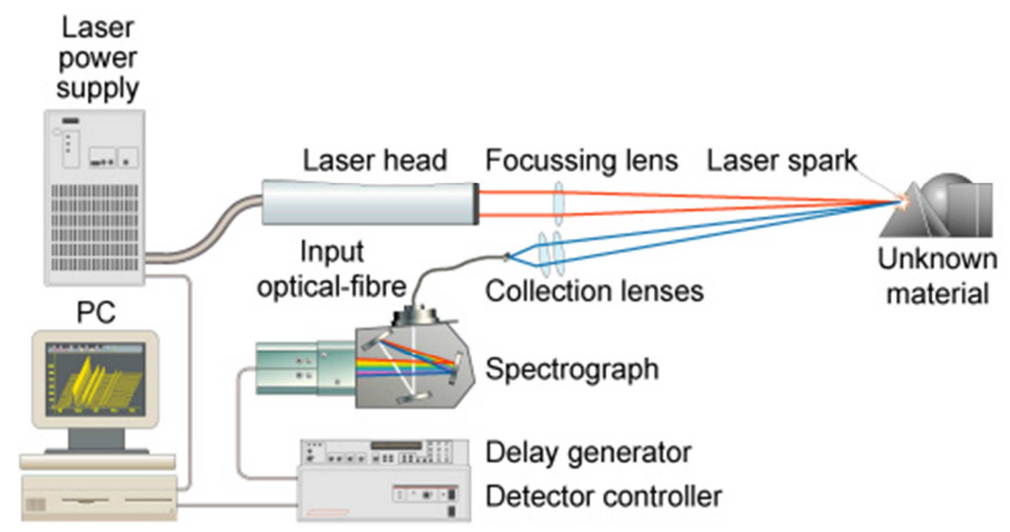

Figure 3. Schematic Representation of Used Setup.

This setup was composed from the following equipments and tools.

\subsubsection{Laser}

Figure 2 shows Q-switched Nd-YAG laser was used here with two wavelengths: $1064 \mathrm{~nm}$ and $532 \mathrm{~nm}$, this Q-switched Nd-YAG laser was used to deliver laser beam at $532 \mathrm{~nm}$, with 20 ns pulse duration and $2 \mathrm{~Hz}$ repetition rate. The pulse energy was $100 \mathrm{mj}$.

\subsubsection{Spectrometer}

To record the emission spectra, a spectrometer type was used. The Ocean Optics USB 4000 Spectrometer is designed from the USB 2000 Spectrometer to include an advanced detector and powerful high-speed electronics to provide both an unusually high spectral response and high optical resolution in a single package the result is a compact, flexible system, with no moving parts, that's easily integrated as an OEM component [12].

The laser to be used is chosen depending on the application, the laser induced breakdown spectroscopy (LIBS) and its main characteristics of using short laser pulses as an energy source to evaporate samples and excite the emission of electromagnetic radiation from its elements and / or molecular fragments, so we use Nd-YAG laser [13].

\subsubsection{Optical Fiber}

The optical fiber used to collect spectra was supplied by Ocean Optics. It is type UV-VIS model Q p 600-2-UV-BX $600 \mu \mathrm{m}$ diameter and two meter length silica core. Surrounding the core is a doped-fluorine silica cladding. A buffer material is then applied. A buffer coats the core and cladding, strengthens the fiber and reduces stray light even further. In most assemblies polyimide is used as the buffer; other assemblies use aluminum or a crylate. Then a jacketing is applied over the, cladding and buffer to protect the fiber and provide strain relief [14].

\subsubsection{Software}

The software (Spectra Suite) used in this work was supplied from Ocean Optics and it is a completely modular, Java-based spectroscopy software plat form that operates on windows, Macintosh and Linux operating systems.
This software can easily manage multiple USB spectrometers each with different acquisition parameters in multiple windows, and provides graphical and numeric representation of spectra from each spectrometer. Using Spectra Suite, one can combine data from multiple sources for applications that include up welling/down welling measurements, dual-beam referencing and process monitoring [15].

\section{Results and Discussion}

LIBS spectrum of sample 1 in the range of (200 to 900) $\mathrm{nm}$ as figure 4 shows.

It shows clear peaks and by comparison with the wavelengths recorded in some references, we found that these wavelengths describe the components of benzene as listed in Table 1.

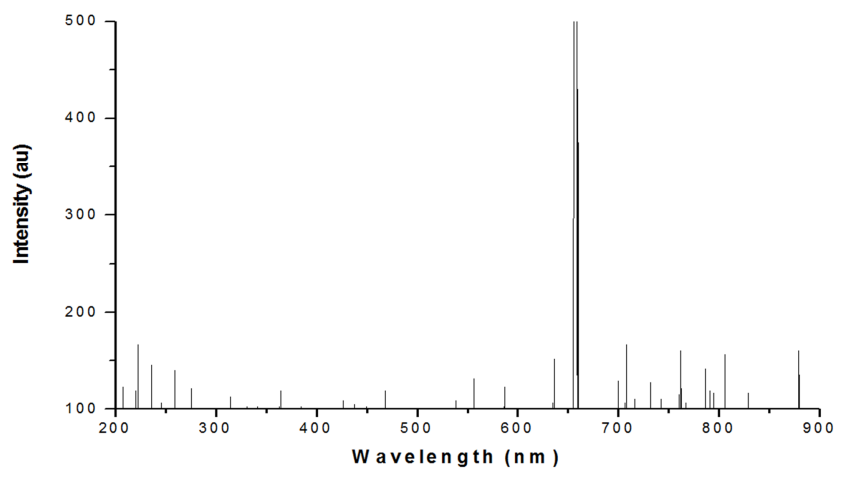

Figure 4. LIBS Spectrum of Benzene Sample No. 1.

Table 1. Analysis of LIBS Emission Spectrum of Sample No 1.

\begin{tabular}{lll}
\hline Wavelength $(\mathbf{n m})$ & Intensity $(\boldsymbol{a} . \boldsymbol{u})$. & Element \\
\hline 210.39 & 124.719101 & $\mathrm{CIV}$ \\
221.83 & 167.41573 & $\mathrm{CIII}$ \\
224.80 & 167.41573 & $\mathrm{WI}$ \\
238.80 & 147.191011 & $\mathrm{PbI}$ \\
248.13 & 108.314607 & $\mathrm{BeI}$ \\
260.48 & 146.516854 & $\mathrm{CII}$ \\
278.56 & 121.797753 & $\mathrm{CoII}$ \\
316.18 & 113.707865 & $\mathrm{CIII}$ \\
366.13 & 127.191011 & HI \\
387.66 & 104.044944 & $\mathrm{CII}$ \\
\hline
\end{tabular}




\begin{tabular}{lll}
\hline Wavelength $(\boldsymbol{n m})$ & Intensity $(\boldsymbol{a} . \boldsymbol{u})$. & Element \\
\hline 428.92 & 109.438202 & TiIII \\
439.73 & 106.741573 & TiIV \\
471.91 & 120.449438 & ZrI \\
541.84 & 109.662921 & KrII \\
559.68 & 133.033708 & SiIII \\
590.08 & 124.494382 & KrII \\
638.37 & 153.483146 & FeII \\
703.00 & 130.11236 & NiI \\
710.01 & 167.191011 & CI \\
719.09 & 112.359551 & HgI \\
720.22 & 112.134831 & CI \\
735.21 & 128.764045 & TiI \\
743.95 & 112.259551 & AgII \\
763.86 & 161.797753 & BII \\
769.20 & 142.47191 & CI \\
789.00 & 120.449438 & SI \\
794.30 & 117.752809 & OI \\
797.77 & 157.752809 & ScI \\
808.60 & 117.752809 & LaI \\
831.42 & 161.797753 & HI \\
882.04 & 108.089888 & OI \\
\hline
\end{tabular}

LIBS spectrum of sample 2 in the range of (100 to 900) $\mathrm{nm}$. Figure 5 shows clear peaks and by comparison with the wavelengths recorded in some references, we found that these wavelengths describe the components of benzene as listed in Table 2.

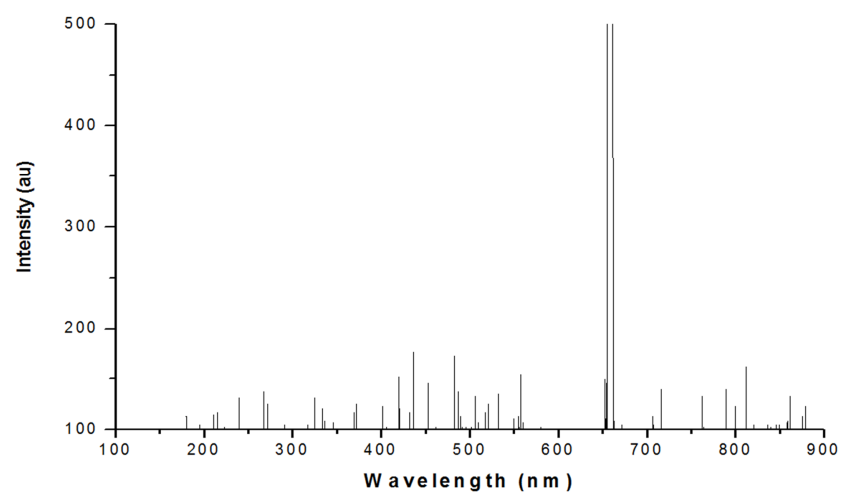

Figure 5. LIBS Spectrum of Benzene Sample No. 2.

Table 2. Analysis of Emission Spectrum of Sample No 2.

\begin{tabular}{lll}
\hline Wavelength $(\mathbf{n m})$ & Intensity $(\mathbf{a u})$ & Element \\
\hline 180.94 & 113.707865 & MnII \\
195.31 & 106.741573 & NeIII \\
209.69 & 116.404494 & ZnI \\
215.72 & 117.752809 & NeIV \\
240.44 & 113.033708 & CIV \\
266.86 & 138.426966 & SII \\
271.20 & 124.719101 & EuIII \\
291.59 & 105.393258 & FeI \\
318.35 & 105.393258 & CII \\
326.36 & 131.685393 & TiII \\
334.72 & 120.674157 & XeII \\
346.76 & 106.741573 & TiI \\
371.50 & 124.719101 & OIII \\
402.25 & 123.370787 & TmI \\
420.63 & 152.134831 & MnII \\
432.67 & 116.404494 & FeI \\
437.13 & 176.853933 & CI \\
453.40 & 145.393258 & YI \\
483.82 & 172.808989 & TiIII \\
\hline
\end{tabular}

\begin{tabular}{lll}
\hline Wavelength $(\boldsymbol{n m})$ & Intensity $(\boldsymbol{a u})$ & Element \\
\hline 488.17 & 137.078652 & CdII \\
506.19 & 133.033708 & KrII \\
520.93 & 124.719101 & ZrI \\
532.96 & 135.730337 & OI \\
549.34 & 111.011236 & FeI \\
557.71 & 153.483146 & EuI \\
672.45 & 105.617978 & CII \\
707.14 & 112.359551 & WI \\
717.17 & 139.775281 & TiIII \\
762.30 & 133.033708 & FeI \\
789.04 & 139.775281 & SI \\
799.07 & 123.370787 & KrI \\
811.44 & 161.797753 & LaIII \\
821.80 & 105.393258 & GdI \\
835.90 & 105.393258 & HI \\
846.70 & 105.393258 & HI \\
850.24 & 105.393258 & HI \\
858.597 & 108.089888 & CII \\
862.60 & 133.033708 & WI \\
877.03 & 112.359551 & CdII \\
878.98 & 123.370787 & GeI \\
\hline
\end{tabular}

Figure 6 shows clear peaks in the range of (200 to 900) nm. By comparison with the wavelengths recorded in some references, we found that these wavelengths describe the components of benzene as listed in Table 3 .

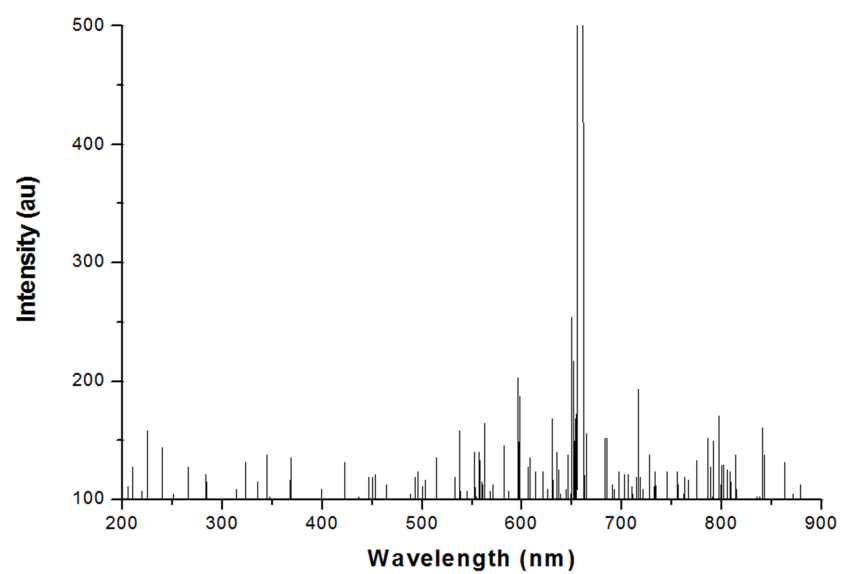

Figure 6. LIBS Spectrum of Benzene Sample No. 3.

Table 3. Analysis of Emission Spectrum of Sample No. 3.

\begin{tabular}{lll}
\hline Wavelength $(\boldsymbol{n m})$ & Intensity $(\boldsymbol{a u})$ & Element \\
\hline 206.89 & 111.011236 & CVI \\
210.39 & 127.415730 & CVI \\
224.22 & 106.741573 & FeII \\
224.73 & 157.752809 & WIII \\
240.82 & 144.044944 & ArII \\
251.64 & 105.393258 & FeII \\
265.97 & 127.415730 & SiII \\
283.82 & 120.674157 & FeII \\
314.24 & 108.314607 & TmI \\
323.31 & 131.460674 & SIII \\
335.89 & 115.05618 & HfI \\
344.66 & 137.078652 & HgI \\
369.71 & 135.730337 & HI \\
400.24 & 108.314607 & TiI \\
423.32 & 131.685393 & OI \\
446.75 & 119.101124 & CeII \\
450.73 & 119.101124 & SI \\
454.06 & 120.449438 & FeI \\
\hline
\end{tabular}




\begin{tabular}{lll}
\hline Wavelength $(\mathbf{n m})$ & Intensity $(\mathbf{a u})$ & Element \\
\hline 464.89 & 112.359551 & NeII \\
493.20 & 119.101124 & CI \\
497.06 & 123.370787 & WI \\
500.60 & 111.011236 & SiI \\
505.21 & 116.404494 & CI \\
514.92 & 135.730337 & FeII \\
532.77 & 119.101124 & NII \\
538.03 & 157.752809 & CI \\
545.34 & 106.741573 & GdI \\
552.65 & 139.775281 & MoI \\
557.92 & 139.775281 & XeI \\
563.19 & 164.494382 & WI \\
570.53 & 112.359551 & TiI \\
583.07 & 145.168538 & VI \\
586.60 & 106.741573 & WI \\
597.42 & 203.146067 & HfI \\
607.95 & 135.730337 & MoI \\
613.50 & 123.370787 & VI \\
620.55 & 1230370787 & CIII \\
631.36 & 168.539326 & NeI \\
634.85 & 139.775281 & FI \\
685.18 & 159.101124 & FeII \\
690.42 & 112.359551 & KrI \\
697.44 & 123.370787 & TiII \\
703.00 & 120.674157 & NiI \\
706.51 & 120.674157 & HeI \\
717.33 & 193.258427 & WI \\
727.87 & 137.078652 & SiII \\
733.45 & 123.370787 & NdI \\
746.00 & 123.370787 & TiIII \\
754.78 & 123.370787 & FeI \\
763.83 & 119.101124 & TiI \\
767.40 & 116.404494 & HgI \\
774.68 & 132.808989 & KrI \\
787.35 & 152.134831 & OIII \\
792.52 & 149.438202 & FeII \\
797.83 & 170.11236 & TiII \\
813.90 & 137.078652 & FeII \\
841.33 & 160.449438 & HI \\
864.26 & 131.685393 & EuI \\
871.26 & 105.393258 & CoII \\
880.38 & 112.359551 & ArII \\
\hline & &
\end{tabular}

Table 4. Comparison between the elements in the three samples.

\begin{tabular}{llll}
\hline \multirow{2}{*}{ Element } & $\begin{array}{l}\text { Sample No. 1 } \\
\text { Intensity (a.u) }\end{array}$ & $\begin{array}{l}\text { Sample No. 2 } \\
\text { Intensity (a.u) }\end{array}$ & $\begin{array}{l}\text { Sample No. 3 } \\
\text { Intensity (a.u) }\end{array}$ \\
\hline $\mathrm{C}$ & 421.744 & 176.853 & 395.258 \\
$\mathrm{H}$ & 288.988 & 316.179 & 296.179 \\
$\mathrm{Fe}$ & 153.483 & 465.846 & 383.595 \\
$\mathrm{Ti}$ & 128.764 & 106.741 & 339.775 \\
$\mathrm{Hg}$ & 112.359 & 0 & 253.483 \\
$\mathrm{Kr}$ & 0 & 123.370 & 245.168 \\
$\mathrm{Eu}$ & 0 & 153.483 & 131.685 \\
$\mathrm{Nd}$ & 0 & 0 & 123.370 \\
$\mathrm{Ni}$ & 130.112 & 0 & 120.674 \\
$\mathrm{He}$ & 0 & 0 & 120.674 \\
$\mathrm{~W}$ & 167.415 & 133.033 & 394.606 \\
$\mathrm{Ne}$ & 0 & 0 & 168.539 \\
$\mathrm{Hf}$ & 0 & 0 & 318.202 \\
$\mathrm{Mo}$ & 0 & 0 & 275.505 \\
$\mathrm{~V}$ & 0 & 0 & 268.539 \\
$\mathrm{Xe}$ & 0 & 0 & 139.775 \\
$\mathrm{Gd}$ & 0 & 105.393 & 106.741 \\
$\mathrm{Si}$ & 0 & 0 & 111.011 \\
$\mathrm{~S}$ & 120.449 & 139.775 & 119.101 \\
$\mathrm{O}$ & 108.089 & 135.730 & 131.685 \\
\hline
\end{tabular}

\begin{tabular}{llll}
\hline \multirow{2}{*}{ Element } & $\begin{array}{l}\text { Sample No. 1 } \\
\text { Intensity (a.u) }\end{array}$ & $\begin{array}{l}\text { Sample No. 2 } \\
\text { Intensity (a.u) }\end{array}$ & $\begin{array}{l}\text { Sample No. 3 } \\
\text { Intensity (a.u) }\end{array}$ \\
\hline $\mathrm{Tm}$ & 0 & 123.370 & 108.314 \\
$\mathrm{Ge}$ & 0 & 123.370 & 0 \\
$\mathrm{Cl}$ & 0 & 108.089 & 0 \\
$\mathrm{Zr}$ & 120.449 & 124.719 & 0 \\
$\mathrm{Y}$ & 0 & 145.393 & 0 \\
$\mathrm{Zn}$ & 0 & 116.404 & 0 \\
$\mathrm{Sc}$ & 157.752 & 0 & 0 \\
$\mathrm{La}$ & 117.752 & 0 & 0 \\
$\mathrm{~Pb}$ & 147.191 & 0 & 0 \\
$\mathrm{Be}$ & 108.314 & 0 & 0 \\
$\mathrm{~F}$ & 0 & 0 & 139.775 \\
\hline
\end{tabular}

Table 5. Comparison between ions in the three samples.

\begin{tabular}{llll}
\hline Ion & $\begin{array}{l}\text { Sample No. 1 } \\
\text { Intensity (a.u) }\end{array}$ & $\begin{array}{l}\text { Sample No. 2 } \\
\text { Intensity (a.u) }\end{array}$ & $\begin{array}{l}\text { Sample No. 3 } \\
\text { Intensity (a.u) }\end{array}$ \\
\hline CIV & 124.719 & 113.033 & 238.426 \\
CIII & 281.123 & 0 & 123.370 \\
CoII & 121.797 & 0 & 105.393 \\
CII & 250.561 & 211.011 & 0 \\
TiIII & 109.438 & 312.584 & 123.370 \\
TiIV & 106.741 & 0 & 0 \\
KrII & 234.157 & 133.033 & 0 \\
SiIII & 133.033 & 0 & 137.078 \\
NeIV & 0 & 117.752 & 0 \\
FeII & 0 & 0 & 659.325 \\
AgII & 112.259 & 0 & 0 \\
BII & 161.797 & 0 & 0 \\
MnII & 0 & 265.842 & 0 \\
NeIII & 0 & 106.741 & 0 \\
SII & 0 & 138.426 & 0 \\
EuIII & 0 & 124.719 & 0 \\
TiII & 0 & 131.685 & 293.483 \\
XeII & 0 & 120.674 & 0 \\
OIII & 0 & 124.719 & 152.134 \\
CdII & 0 & 249.438 & 0 \\
LaIII & 0 & 161.797 & 0 \\
WIII & 0 & 0 & 157.752 \\
ArII & 0 & 0 & 256.404 \\
SiII & 0 & 0 & 127.415 \\
SIII & 0 & 0 & 131.460 \\
CeII & 0 & 0 & 119.101 \\
NeII & 0 & 0 & 112.359 \\
NII & 0 & 0 & 119.101 \\
\hline & & & \\
\hline & 0 & 0 & 0 \\
\hline
\end{tabular}

From the results obtained in this research one can conclude that:

The essential atoms forming the samples of benzene (Hydrogen and Carbon) appeared with great amounts while other elements such as (Pb, Be, Zr, Ni, Hg, Ti, Sc, La, Zn, Tm, Y, $\mathrm{Eu}, \mathrm{Kr}, \mathrm{Gd}, \mathrm{Cl}, \mathrm{Ge}, \mathrm{Hf}, \mathrm{Mo}, \mathrm{Xe}, \mathrm{V}, \mathrm{Mo}, \mathrm{Ne}, \mathrm{He}$ and $\mathrm{Nd}$ ) were appeared with little amounts relative to the essential atoms. Also, these elements were found in varying quantities in the three samples, as the figure 4 shows. Some of them were found in the three samples and some were found in two samples and there was not found in the third sample and some of them were found in one sample only, and this is due to the different regions from which the samples were collected.

(C, H. Fe, Ti, W, O and S) atoms were found in the three samples with different amounts.

Some of the elements that have been discovered in benzene samples such as $(\mathrm{Pb}, \mathrm{Hg}$ and $\mathrm{Zn})$ are among the elements that may cause extensive environmental damage at 
the human (some of which cause cancer, such as lead), animals, and plants.

Note that the samples contain lead, which is the first to have the type of toxicity. Lead has been criminalized in a wide range of toxic effects and is considered a durable heavy metal ubiquitous. Exposure to this element may harm humans and cause diseases such as cancer and affect the functions of the kidneys, liver and lungs, as well as in wildlife. The lead balance is a real threat to public health, especially in developing countries. Therefore, the competent authorities in Sudan must solve this problem and make the benzene unleaded or reduce its quantity as a minimum [16].

Among the group of contaminants, $\mathrm{Hg}$ toxicity is considered to be an important contributor to the overall health risk presented by contaminants in the environment.

Mercury is a toxic and non-essential metal in the human body. Inorganic mercury compounds are water soluble with a bioavailability of $7 \%$ to $15 \%$ after ingestion; they are also irritants and cause gastrointestinal symptoms. Upon entering the body, inorganic mercury compounds are accumulated mainly in the kidneys and produce kidney damage. In contrast, human exposure to elemental mercury is mainly by inhalation, followed by rapid absorption and distribution in all major organs. Elemental mercury from ingestion is poorly absorbed with a bioavailability of less than $0.01 \%$. The primary target organs of elemental mercury are the brain and kidney. Elemental mercury is lipid soluble and can cross the blood-brain barrier, while inorganic mercury compounds are not lipid soluble, rendering them unable to cross the bloodbrain barrier. Elemental mercury may also enter the brain from the nasal cavity through the olfactory pathway. The blood mercury is a useful biomarker after short-term and high-level exposure, whereas the urine mercury is the ideal biomarker for long-term exposure to both elemental and inorganic mercury, and also as a good indicator of body burden [17].

\section{Conclusions}

The laser induced breakdown spectroscopy (LIBS) technique is very accurate. LIBS is capable to detect almost all the elements and ions in the benzene samples and can be used as diagnostic technique for investigation of elements in liquid samples.

\section{Recommendations}

From the results, the followings can be suggested as future work:

Improving the quality of benzene in Sudan by eliminating the toxic elements $(\mathrm{Pb}, \mathrm{Hg}$ and $\mathrm{Zn})$.

LIBS method can be considered in the future for analysis of many classes of organic compounds.

Diode pulse lasers can be used to overcome the laser flash lamp background.

Study the percentage of the heavy elements in the benzene. Study the effect of the addition of chemical improvements on the benzene that used in Sudan.

\section{Acknowledgements}

We wish to thank the Institute of Laser in Sudan for supporting this research for recording the LIBS spectra.

\section{References}

[1] Duarte, F. J. ed., 2008. Tunable laser applications (Vol. 150). CRC press.

[2] Rencz, A. N. and Ryerson, R. A. eds., 1999. Manual of remote sensing, remote sensing for the earth sciences (Vol. 3). John Wiley \& Sons.

[3] Ciucci, A., Palleschi, V., Rastelli, S., Barbini, R., Colao, F., Fantoni, R., Palucci, A., Ribezzo, S. and Van der Steen, H. J. L., 1996. Trace pollutants analysis in soil by a time-resolved laser-induced breakdown spectroscopy technique. Applied Physics B, 63 (2), pp. 185-190.

[4] Singh, J. P. and Thakur, S. N. eds., 2020. Laser-induced breakdown spectroscopy. Elsevier.

[5] HAIDER, Z., MUNAJAT, Y. and KAMARULZAMAN, R., 2012. (LIBS) A PROMISING TECHNIQUE, ITS LIMITATIONS AND A PROPOSED METHOD. Jurnal Teknologi, 57 (1).

[6] Kasper, Dennis L. et al. 2004 Harrison's Principles of Internal Medicine, 16th ed., McGraw-Hill Professional, p. 618, ISBN 0071402357.

[7] Bard, D., 2014. Traffic-related air pollution and the onset of myocardial infarction: disclosing benzene as a trigger? A small-area case-crossover study. PLOS ONE. 9 (6): 6. Bib code: $2014 \quad$ PLoSO...9j0307B. doi: 10.1371/journal.pone.0100307. PMC 4059738. PMID 24932584.

[8] Smith, Martyn T. 2010. Advances in understanding benzene health effects and susceptibility. Annu Rev Public Health. 31: 133-48. doi: 10.1146/annurev.publhealth.012809.103646. PMC 4360999. PMID 20070208.

[9] Cao, L., Jiang, H., Yang, J., Fan, L., Li, F. and Huang, Q., 2013. Simultaneous determination of benzene and toluene in pesticide emulsifiable concentrate by headspace GC-MS. Journal of analytical methods in chemistry, 2013.

[10] WHO. International Agency for Research on Cancer, IARC Monographs on the Evaluation of Carcinogenic Risks to Humans, Overall Evaluations of Carcinogenicity: An Updating of IARC Monographs Archived 2008-03-06 at the Wayback Machine, Volumes 1 to 42, Supplement 7.

[11] Rana, S. V. and Verma, Y., 2005. Biochemical toxicity of benzene. Journal of Environmental Biology, 26 (2), pp. 157168 .

[12] http://www.ocean optics.com/product/ USB4000 UV/VIB. Retrieved at July3013.

[13] Pasquini, C., Cortez, J., Silva, L. and Gonzaga, F. B., 2007. Laser induced breakdown spectroscopy. Journal of the Brazilian Chemical Society, 18 (3), pp. 463-512. 
[14] http://sciencehowstuffworks.com/laseranalysis.htm.RetrievedatJuly2013.

[15] Werdell, J., 2009. Global Bio-optical Algorithms for Ocean Color Satellite Applications: Inherent Optical Properties Algorithm Workshop at Ocean Optics XIX; Barga, Italy, 3-4 October 2008. Eos, Transactions American Geophysical Union, 90 (1), pp. 4-4.
[16] Assi, M. A., Hezmee, M. N. M., Abd Wahid Haron, M. Y. M. and Sabri, M. A. R., 2016. The detrimental effects of lead on human and animal health. Veterinary world, 9 (6), p. 660.

[17] Park, J. D. and Zheng, W., 2012. Human exposure and health effects of inorganic and elemental mercury. Journal of preventive medicine and public health, 45 (6), p. 344. 